\title{
Gene delivery of a single,structurally engineered Coronavirus vaccine antigen elicits SARS-CoV-2 Omicron and pan-Sarbecovirus neutralisation
}

Jonathan Heeney ( $\sim$ jlh66@cam.ac.uk)

University of Cambridge https://orcid.org/0000-0003-2702-1621

Sneha Vishwanath

University of Cambridge

George Carnell

University of Cambridge https://orcid.org/0000-0001-8875-0989

Matteo Ferrari

DIOSynVax Ltd

\section{Benedikt Asbach}

University of Regensburg https://orcid.org/0000-0003-1056-8591

Martina Billmeier

University of Regensburg

Charlotte George

University of Cambridge https://orcid.org/0000-0002-6585-3288

Maria Suau Sans

University of Cambridge

Patrick Neckermann

University of Regensburg

David Peterhoff

University of Regensburg

\section{Diego Cantoni}

Viral Pseudotype Unit, Medway School of Pharmacy, University of Kent

David Wells

Liverpool John Moores University https://orcid.org/0000-0002-4531-5968

Nigel Temperton

University of Kent https://orcid.org/0000-0002-7978-3815

Simon Frost

Microsoft Health Futures 
Keywords:

Posted Date: January 26th, 2022

DOI: https://doi.org/10.21203/rs.3.rs-995273/v1

License: (c) (1) This work is licensed under a Creative Commons Attribution 4.0 International License. Read Full License 
Gene delivery of a single, structurally engineered Coronavirus vaccine antigen elicits pan-

4 DBPR

5 


\section{$6 \quad$ Abstract}

7 Of the coronaviruses that have caused zoonotic spill-overs in past two decades, the diverse

8 group of betacoronaviruses (B-CoVs) represent the greatest threats. Towards achieving broad

9 vaccine protection from these viruses, vaccines composed of multiple antigens, each capable

10 of eliciting broad neutralising responses across a subgroup will be required. Here we describe

11 a novel platform for selecting immune optimized, structurally engineered antigens capable of

12 eliciting protective responses across a group of related viruses and demonstrate proof-of-

13 concept against the sarbecoviruses subgenus with a single antigen structure. From an array of

14 phylogenetically informed antigen structures displaying different broad neutralising epitopes,

15 synthetic genes expressing these were selected based on broad immune responses in BALB/C

16 mice. Improved protection against the Delta variant was observed in K18-hACE2 mice on

17 boosting with the lead designs of mice primed by an approved COVID-19 vaccine.

18 Immunogenicity of the lead vaccine antigen was confirmed in guinea pigs using needleless

19 intradermal immunisation. The broad neutralising immune profile against SARS-CoV, SARS-

20 CoV-2, WIV16, and RaTG13 was further confirmed in rabbits with GMP manufactured DNA

21 immunogen. Notably, sera from immunised rabbits showed potent antibody responses against

22 Beta, Gamma, and Delta variants of concern. Here we demonstrate proof-of-concept of this

23 Digitally Immune Optimised, Selected vaccine (DIOSvax) antigen pipeline for the in vivo

24 selection of single nucleic acid-based immunogens. Such gene-based antigens can be readily

25 delivered alone or, in combination for vaccines designed to prevent future pandemics, can be

26 seamlessly scaled with vaccine delivery modalities such as viral vector or mRNA based 27 vaccines. 
30 Amongst the coronaviruses of the greatest pandemic risk are the angiotensin-converting enzyme 2 (ACE-2) binding viruses of $\beta$-Coronaviruses genus ${ }^{1,2}$. Over the last two decades, two ACE-2 binding sarbecoviruses (a sub-genus of $\beta$-coronaviruses) have spilled over into human population causing the SARS epidemic in 2002/2003 and the current on-going SARSCoV-2 pandemic. Bats are the reservoir of a large number of SARS-CoV-like ACE-2 binding sarbecoviruses which pose a constant threat for future spill-over into human population, and potentially new epidemics ${ }^{3,4}$. In addition to emergence of new ACE-2 binding viruses from zoonotic reservoirs, another concern is the emergence of variants of these viruses capable of escaping vaccine-induced immunity, a constant concern in the current on-going pandemic. As human infections increase globally during the current pandemic, the virus has continued to accrue mutations, most significantly in the spike protein ${ }^{5}$. An accumulating number of variants of concern (VOCs) have implications for increased transmission and escape from natural and vaccine immunity ${ }^{6-9}$. The N501Y asparagine to tyrosine substitution in the receptor binding domain (RBD) of the spike protein is a common feature of VOCs and is associated with increased affinity of the viral spike protein to the ACE- 2 receptor and subsequent increase in transmission ${ }^{10}$. To date, two variants have K417N/T and E484K mutations in the RBD and are reported to escape immune responses generated by most approved vaccines ${ }^{7,8}$. The Delta

$47 \mathrm{VOC}^{11}$ is the most contagious variant reported to date, with L452R and T478K mutations in the RBD. Notably, the majority of these mutations reported in VOCs are in or around the region in RBD that interacts with ACE-2 as well as one of the regions that induce highly potent neutralising antibodies ${ }^{12,13}$. The continued emergence of these VOCs during the on-going COVID-19 pandemic, and the constant threat of new zoonotic spillovers of coronaviruses from animals to humans, highlights the need for next generation vaccines with broader protection from ACE-2 binding sarbecoviruses as well as the emerging VOCs. To increase the coverage 
to all ACE-2 receptor using viruses of the sarbecovirus sub-genus of $\beta$-coronaviruses, we utilised a computational structure-based, RBD subunit-based vaccine strategy comparing all the known human and animal reservoir sarbecoviruses. This design was further used as backbone for designing both epitope optimised and immune re-focussed designs using available structural data for spike protein in complex with monoclonal antibodies, specifically those targeting the ACE-2 receptor binding domain (RBD), such as $\mathrm{S} 309^{14}$ and CR3022 $2^{15}$ that bind both SARS-CoV, and SARS-CoV-2. The nucleic acid sequence of these in silico designed vaccine antigens were optimised for expression in humans and synthetic genes expressing each unique antigen structure was shuttled in an expression cassette for consecutive in vitro and in vivo screens in different species to select the best-in-class immunologically optimal antigen as the vaccine candidate for nucleic acid vaccine delivery.

Sequences of spike protein of viruses belonging to the sarbecovirus lineage were compiled from NCBI virus database ${ }^{16}$ and further pruned. The phylogenetic tree of these sequences is represented in Fig. 1A. Two distinct clades are observed in the tree, separating those in clade 1, which do not interact with ACE-2 receptor $^{1,17}$ from those in clade 2 , which do. Clade 1 viruses share many of the sequence features of the members of clade 2 but possess deletions around the ACE-2 binding region (Fig. S1). An optimised core sequence (T2_13) was designed, such that each amino acid position in this sequence was optimised to be phylogenetically closer to all the sarbecoviruses represented in the phylogenetic tree in Fig. 1A. To further understand the importance of amino-acid composition of epitopes in generating antibody responses, we further modified T2_13 to display the epitopes of SARS-CoV for monoclonal antibodies - S30914 (T2_14), and CR3022 ${ }^{15}$ (T2_15) and of SARS-CoV-2 for monoclonal antibody - B38 ${ }^{12}$ (T2_16). The sequence of epitopes for monoclonal antibodies $\mathrm{S} 309^{14}$, and CR3022 $2^{15}$ are highly conserved across the sequences considered in this study while 
the sequence of epitopes for monoclonal antibody - B38 $8^{12}$ is highly divergent (Fig. 1B). We further modified the epitope region for monoclonal antibody - B38 ${ }^{12}$ by introducing a glycosylation site on the backbone of T2_14 (T2_17) and T2_15 (T2_16). This was done to mask the divergent epitope region and enhance the presentation of the conserved epitopes to the immune system. The masking of epitopes by introducing glycans has been exploited by many viruses such as Hepatitis $\mathrm{C}$ Virus ${ }^{18}$, Lassa virus ${ }^{19}$, and Influenza ${ }^{20}$ to escape natural immunity. To compare the immunogenicity of soluble and membrane bound RBD subunitbased vaccine, membrane bound forms of T2_13 and T2_17 (T2_13_TM and T2_17_TM respectively) were generated. The structural stability of these designs was evaluated in-silico using the FOLDX ${ }^{21}$ algorithm using T2_13 as the reference model. Structural models of these vaccine antigens are represented in Fig. 1C.

In vivo screening in $\mathrm{BALB} / \mathrm{c}$ mice was performed by immunising with different lead antigen designs (Fig. 1D) and assaying for cross reactive antibodies against different sarbecovirus spike proteins in a flow cytometry based cell-surface display assay. Sera taken two weeks following the second immunisation with antigen designs (T2_13 through to T2_18 and the membrane bound designs), demonstrated the binding profile of the vaccine candidates for different spike proteins (Fig. 1E). As expected, sera from SARS-CoV RBD immunised mice bound strongly to both homologous SARS-CoV spike protein and closely related WIV16 spike protein in comparison to other vaccine designs, while sera from SARS-CoV-2 RBD immunised mice bound homologous SARS-CoV-2 spike protein and closely related RaTG13 spike protein in the similar range of other vaccine designs. Sera from SARS-CoV immunised mice showed binding to SARS-CoV-2 spike, though significantly less than sera from SARS-CoV-2 RBD 102 immunised mice $(\mathrm{p}=0.02)$ and comparable to PBS $(\mathrm{p}>0.05)$. Across the four spike proteins, no significant differences in binding were observed for sera from mice immunised with $\mathrm{T} 2 \_13$ 
and sera from mice immunised with SARS-CoV-2 RBD (all $p>0.05$ ), demonstrating that epitopes in this design is biased towards SARS-CoV-2 RBD. For the T2_16 design, in which the epitope region for $\mathrm{mAb} \mathrm{B} 38$ was mutated to epitope region on SARS-CoV-2, binding to SARS-CoV, WIV16, and RaTG13 declined in comparison to T2_13 ( $p<0.05$ ) without statistical changes in binding to SARS-CoV-2. This observation is suggestive of immunodominance of this region in comparison to other sites. Matching of the epitopes of S309 and CR3022 to SARS-CoV (T2_14 and T2_15), enhanced the binding to SARS-CoV (p

$111<0.05)$ but not to other spike proteins. Introduction of glycosylation site in design T2_17

112 significantly enhanced the binding of elicited antibodies to SARS-CoV and RaTG13 $(\mathrm{p}<0.01)$

113 in comparison to T2_14, but no difference was observed in T2_18 in comparison to T2_15.

114 There was no difference between trans-membrane and non- trans-membrane bound designs.

115 As T2_17 has either the best (or second best) median binding to the four spike proteins apart

116 from the homologous RBD vaccine antigen, we choose T2_17 as the lead candidate for further 117 immunological assays.

119 Elicitation of cross-binding antibodies by T2_17 was further confirmed by ELISA with SARS120 CoV RBD and SARS-CoV-2 RBD (Fig. 1F), revealing robust binding antibody responses to both SARS-CoV and SARS-CoV-2 within two weeks of the second immunisation. While the T2_17 antigen elicited stronger responses against SARS-CoV, it was lower than those induced by the homologous SARS-CoV antigen, but significantly higher than SARS-CoV-2. Against SARS-CoV-2, all the three antigens - SARS-CoV RBD, SARS-CoV-2 RBD, and T2_17 generated similar binding antibody responses. Given the breadth of antibody responses induced by the T2_17 antigen, we asked if this antigen could boost and broaden the efficacy of current

127 licensed vaccines against SARS-CoV-2 VOCs. To address this, we used homozygous K18128 hACE2 transgenic mice and immunised them with $1.4 \times 10^{9} \mathrm{vp}$ of commercially available 
129 AZD1222 (ChAdOx1 nCoV-19) and 4 weeks later boosted with either T2_17, or the licensed

130 AZD1222 vaccine (Fig. 1G), while the control group received only PBS with each

131 immunisation. Eight weeks post boost, all groups of mice were challenged with either a January

1322020 isolate of SARS-CoV-2 (Victoria) or the more recent Delta variant of SARS-CoV-2 (Fig.

133 1H). Increased binding antibodies titres to both SARS-CoV and SARS-CoV-2 after boosting

134 by either AZD1222 or T2_17 (Fig. S2) was observed. Significant difference in antibody titres

135 to SARS-CoV-2 were observed four weeks after boosting with T2_17 in comparison to

136 boosting by AZD1222 (Fig. 1I). Neutralising antibodies for SARS-CoV-2 and the Delta VOC

137 were detected for all the groups, except the control group prior to challenge (Fig. S3). After

138 two weeks post boost, T2_17 neutralised the Delta variant significantly better than the sera

139 from mice boosted with AZD1222 (Fig. 1J). Mice from all the groups, except controls,

140 survived and continued to gain weight following challenge with either the Victoria strain or

141 Delta variant (Fig. 1K).

142 

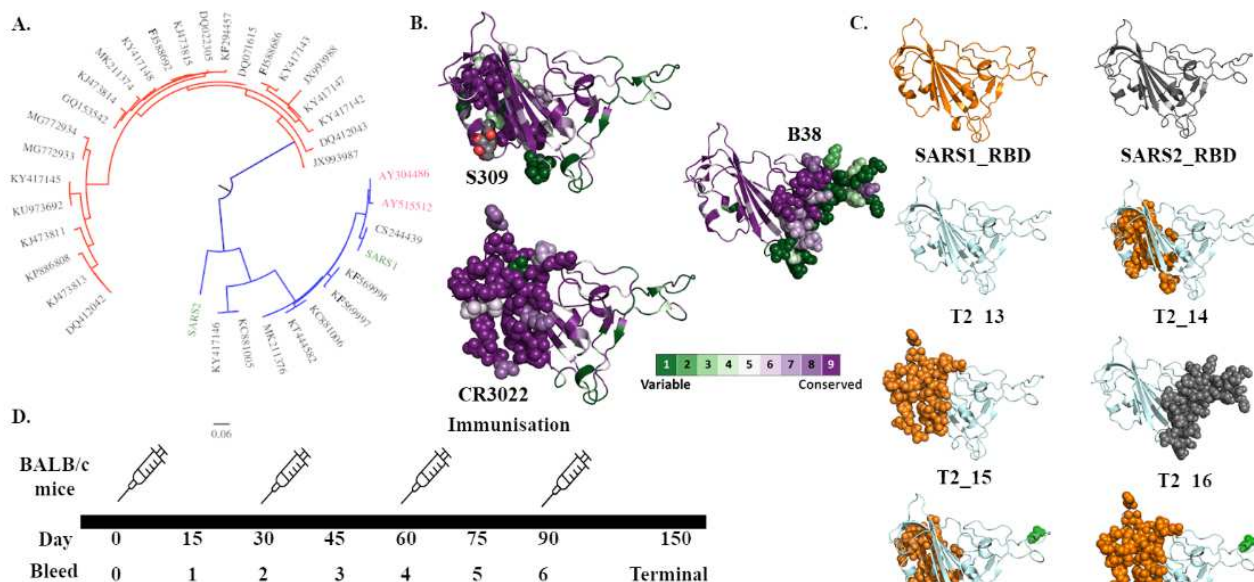
SARS1_RB

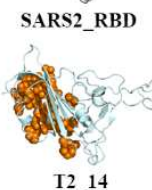

T2 13
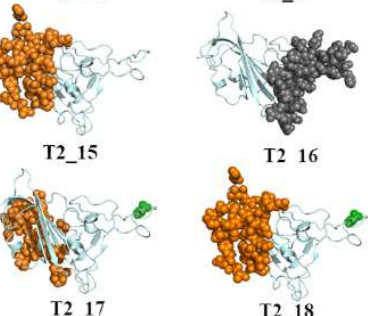

12 -17
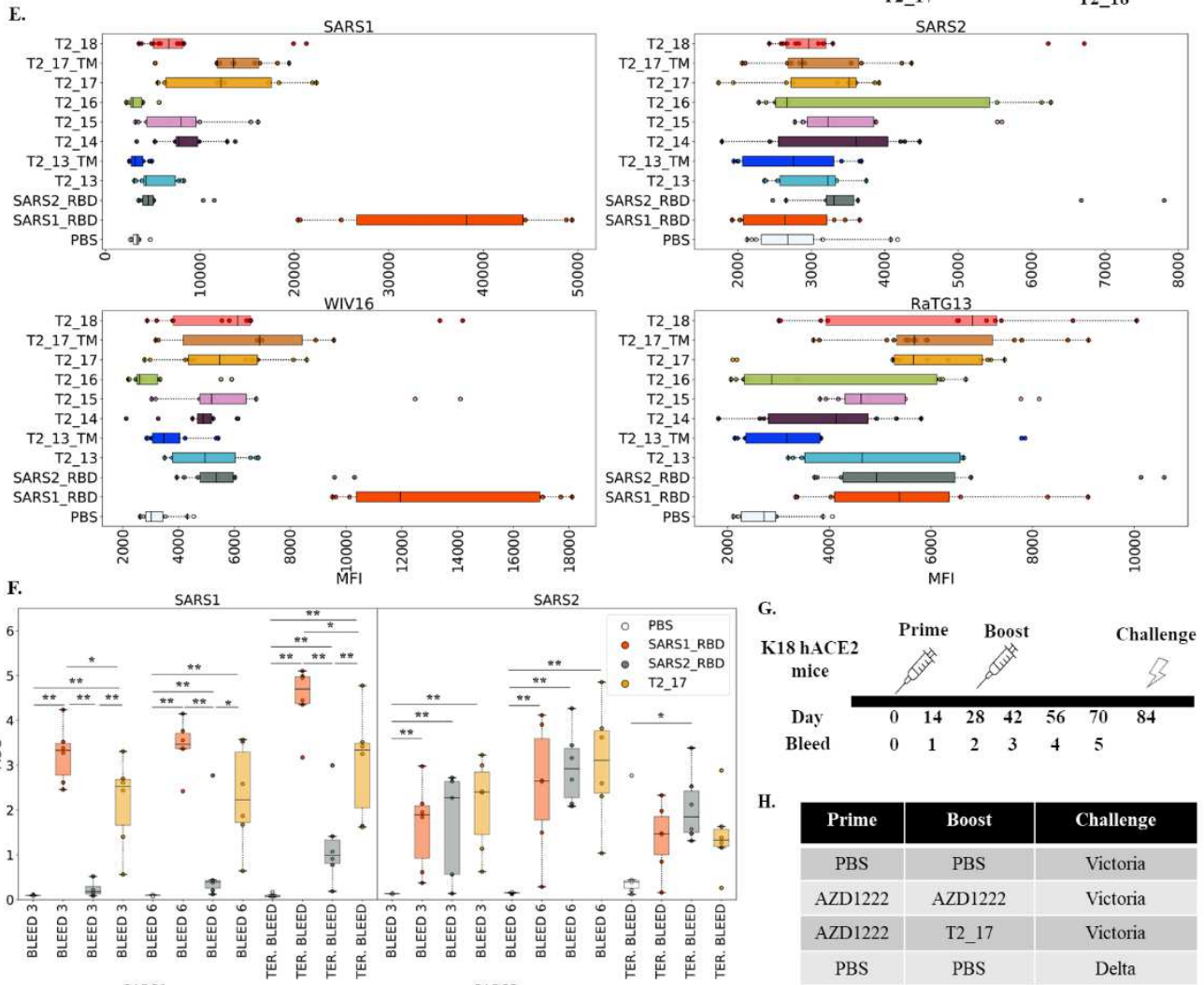

$\begin{array}{lllllll}0 & 1 & 2 & 3 & 4 & 5\end{array}$

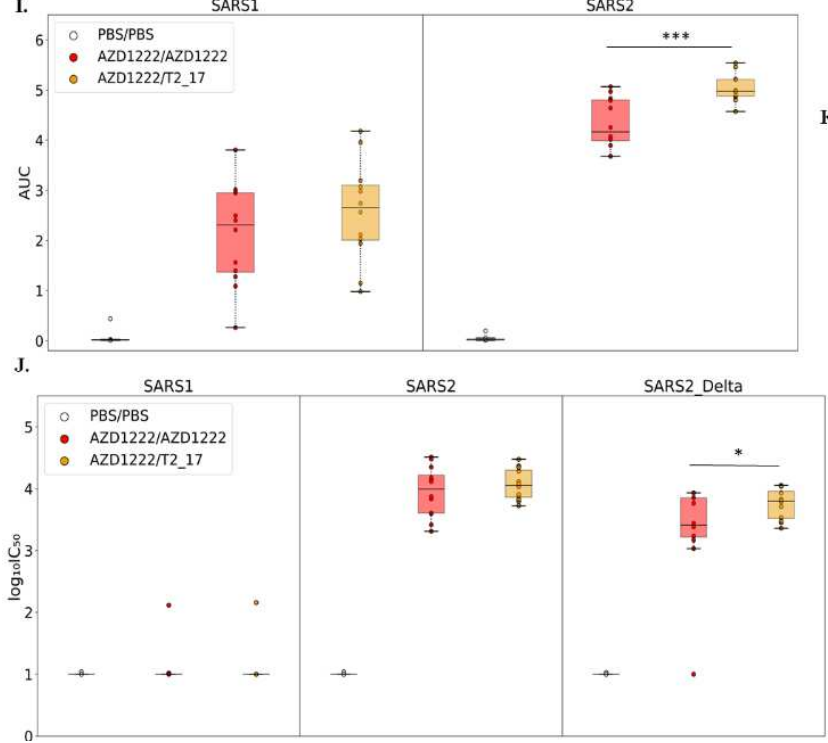

\begin{tabular}{|c|c|r|}
\hline Prime & Boost & Challenge \\
\hline PBS & PBS & Victoria \\
\hline AZD1222 & AZD1222 & Victoria \\
\hline AZD1222 & T2_17 & Victoria \\
\hline PBS & PBS & Delta \\
\hline AZD1222 & AZD1222 & Delta \\
\hline AZD1222 & T2_17 & Delta \\
\hline
\end{tabular}
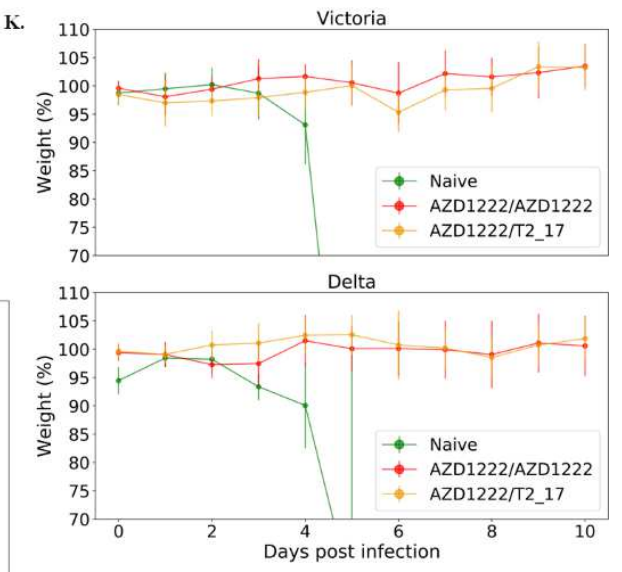
145 A. Phylogenetic tree generated for sarbecoviruses using protein sequence of receptor binding domain (RBD) of the spike protein. The tree was generated using IQ-Tree ${ }^{22}$. Human viruses are represented in green, palm civet viruses in pink and bat viruses in dark grey. The distinct two clades are coloured in red (non-ACE-2 binding) and blue (ACE-2 binding). B. Structural models of RBD with epitope regions highlighted as spheres. The backbone of RBD is coloured according to the CONSURF ${ }^{23}$ score calculated using the alignment used for construction of phylogenetic tree. The figure was generated and rendered using $\mathrm{PyMol}^{24}$ using $\mathrm{PDB}^{25}$ id $6 \mathrm{wps}^{14}, 6 \mathrm{w} 41^{15}$, and $7 \mathrm{bz} 5^{12}$. C. Structural representation of the different vaccine designs used in this study. The epitopes that were modified to match the wild-type SARS-CoV (coloured orange) and wild-type SARS-CoV-2 (coloured grey) are represented in spheres. Further glycosylation site modification is represented in green sphere. D. Immunisation and bleed schedule of BALB/c mice. Mice were immunised at interval of 30 days and bled every 15 days. E. FACS binding data for different vaccine designs. Sera from mice immunised with these vaccine antigens were screened for binding to SARS-CoV, SARS-CoV-2, WIV16, and RaTG13 spike proteins. The X-axis represents the mean fluorescence intensity (MFI), and the $\mathrm{Y}$-axis represents all the vaccine designs considered for screening. For each mouse sera, two replicates of MFI have been reported. F. Elicitation of binding anti-bodies against SARS-CoV and SARS-CoV-2 by T2_17 was confirmed using ELISA, with SARS-CoV and SARS-CoV2 RBD as control vaccine design. T2_17 generated cross-binding antibodies. The $\mathrm{X}$-axis represents the vaccine designs, and the $\mathrm{Y}$-axis represents the area under the curve (AUC) for ELISA binding curves. G. Immunisation, bleed, and challenge schedule of K18-hACE2 mice. H. K18-hACE2 mice were primed with AZD1222 vaccine and then boosted with either AZD1222, or T2_17 after four weeks. The mice were challenged after 8 weeks with either Victoria strain of SARS-CoV-2 or the Delta variant. I. Elicitation of binding antibodies against SARS-CoV and SARS-CoV-2 before challenge was confirmed using ELISA using K18hACE2 mice sera 4 weeks post boost (bleed4). Boost by T2_17 significantly increased the binding antibody titres in comparison to boost by AZD1222. The X-axis represents the vaccine designs, and the $\mathrm{Y}$-axis represents the area under the curve (AUC) for ELISA binding curves for each serum. J. Neutralisation of SARS-CoV, SARS-CoV-2, and delta variant of SARSCoV-2 by K18-hACE2 mice sera 4 weeks post boost (bleed4). Sera of mice boosted with T2_17 significantly neutralised the Delta variant (B.1.617.2) in comparison to those boosted by AZD1222. The X-axis represents the bleed number, and the $\mathrm{Y}$-axis represents the $\log _{10} \mathrm{IC}_{50}$ values for neutralisation curves. K. Weight loss profile of K18-hACE2 mice following challenge by the Victoria strain and the Delta variant. All the mice, except naïve were protected. Mann-Whitney $U$ test is used as statistical significance test in all the plots (p-value: ${ }^{*} \leq 0.05$, $* *<0.01, * * * \leq 0.001)$.

To determine the breadth of antibody response and neutralisation in outbred animals, guinea 
delivery (Fig. 2A). As a control we used a C-terminal glycosylation modified SARS-CoV-2 RBD (SARS2_RBD_P521N) (Fig. 2B) which we had previously evaluated in BALB/c mice

187 (Fig. S4). Generation of neutralising antibodies to both SARS-CoV and SARS-CoV-2 was confirmed using pseudoviruses expressing full-length spike proteins of SARS-CoV, and SARS-CoV-2. While both T2_17 and SARS2_RBD_P521N generated binding antibodies against both SARS-CoV and SARS-CoV-2 (Fig. 2C) after one immunisation, T2_17 elicited significantly higher antibodies than SARS2_RBD_P521N to SARS-CoV and comparable antibodies against SARS-CoV-2. Higher binding antibodies were detected for T2_17 to SARSCoV in comparison to SARS2_RBD_P521N after two immunisations while the responses were comparable for SARS-CoV-2. After three immunisations SARS2_RBD_P521N induced a higher response to SARS-CoV-2, while T2_17 had higher responses to SARS-CoV. Neutralising antibodies were detected for SARS-CoV-2 after first immunisation, while significant neutralising responses to SARS-CoV developed after two immunisations, though more potent for T2_17 than SARS2_RBD_P521N (Fig. 2D). Better binding and neutralising responses by SARS2_RBD_P521N to SARS-CoV-2 were expected as it differs from SARS-

200 CoV-2 by only one amino acid. To further confirm, whether T2_17 vaccine design generates broader responses, we compared sera induced by SARS2_RBD_P521N, 28 days post 3rd immunisation for neutralisation against SARS-CoV, WIV16, RaTG13, and SARS-CoV-2. Statistically significant higher titre neutralising antibodies were generated by T2_17 against SARS-CoV, WIV16, and RaTG13 (Fig. 2E). To further confirm T2_17 anti-sera could abrogate hACE2 receptor binding, we performed an ELISA based competition assay (Fig. 2F) demonstrating T2_17 and SARS2_RBD_P521N anti-sera abrogated binding to hACE-2 receptor and are comparable to the WHO standard of pooled convalescent COVID-19 patient sera. These findings demonstrated important proof-of-concept of T2_17 as a single gene delivered, structurally engineered antigen capable of eliciting broad pan-sarbeco Coronavirus 
210 neutralising antibodies. Prior to clinical trials in humans, a GMP lot of pEVAC T2_17 was

211 manufactured and evaluated for safety and immunogenicity in rabbits using the same gene

212 delivery device to ensure uniform intradermal administration (Fig. 2G). After one

213 immunisation, binding antibodies to SARS-CoV and SARS-CoV-2 were elicited (Fig. 2H),

214 increasing on subsequent immunisations until a plateau was reached by the fourth

215 immunisation. Robust neutralising antibodies were observed 2 weeks following the third

216 immunisation (Fig. 2I) revealing broad neutralising antibody responses against the SARS-

217 CoV, SARS-CoV-2, Beta, Gamma, and Delta VOCs as well as the bat sarbecoviruses -

218 WIV16, and RaTG13 elicited by gene delivery of the engineered T2_17 pan-Sarbeco vaccine

219 antigen candidate (Fig. 2J).

220 

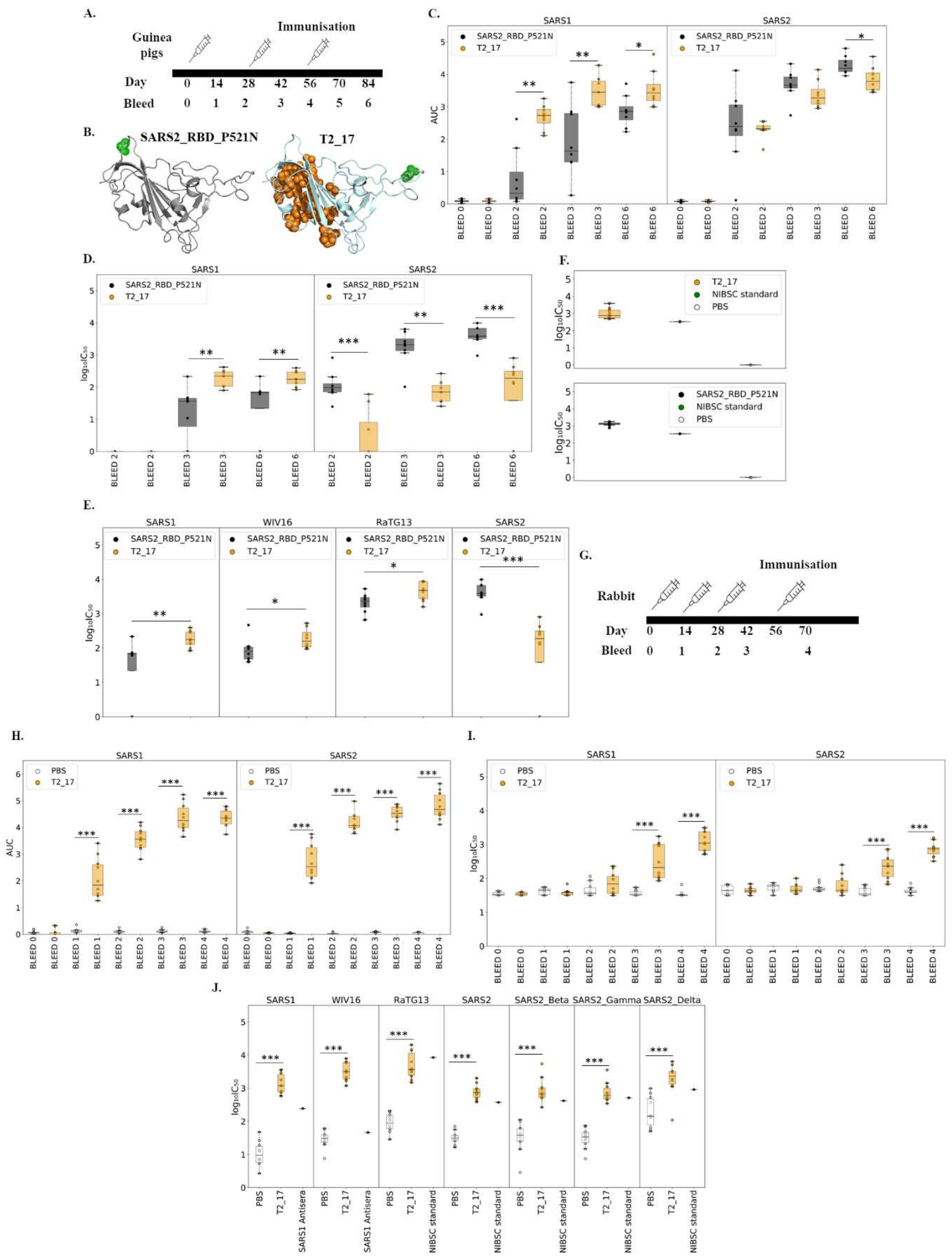

Figure 2| Immunogenicity studies in guinea pigs and rabbits

223 A. Immunisation and bleed schedule of guinea pigs. Guinea pigs were immunised with DNA 224 delivered intradermally (i.d) by the Tropis ParmaJet device at 28 day intervals and bled every 22514 days. B. Structure models of the vaccine designs used for the study in guinea pigs. The 226 glycosylation site and the modified epitope are represented as green and orange spheres 
228 T2_17 and SARS2_RBD_P521N was confirmed using ELISA. T2_17 and 229 SARS2_RBD_P521N generated cross-binding antibodies after one immunisation. The pre230 bleed (Bleed 0) is considered as the control for non-specific binding. The X-axis represents the bleed number, and the Y-axis represents the area under the curve (AUC) for ELISA binding curve. D. Neutralisation by guinea pig sera immunised with T2_17 and SARS2_RBD_P521N. Both T2_17 and SARS2_RBD_P521N generated neutralising antibodies against SARS-CoV and SARS-CoV-2. The X-axis represents the bleed number, and the $\mathrm{Y}$-axis represents the $\log _{10} \mathrm{IC}_{50}$ values for neutralisation curves. E. Broad-neutralisation of SARS-CoV, WIV16, RaTG13, and SARS-CoV-2 by T2_17 in comparison to SARS2_RBD_P521N. Sera post 28 days after three immunisation (bleed 6) was used for comparison. F. ACE-2 competition ELISA. Sera from Guinea pigs immunised with T2_17 and SARS2_RBD_P521N effectively abrogated the interaction of SARS-CoV-2 RBD with ACE-2 receptor. The NIBSC standard (20/162) was used as control. G. Immunisation and bleed schedule of Rabbits. Rabbits were immunised at interval of 14 days and bled every 14 days. H. Elicitation of binding antibodies against SARS-CoV and SARS-CoV-2 by T2_17 was confirmed using ELISA. T2_17 generated cross-binding antibodies after one immunisation. The $\mathrm{X}$-axis represents the bleed number, and the $\mathrm{Y}$-axis represents the area under the curve (AUC) for ELISA binding curve. I. Neutralisation by rabbit sera immunised with T2_17. T2_17 generated neutralising antibodies against SARS-CoV and SARS-CoV-2. The X-axis represents the bleed number, and the $\mathrm{Y}$-axis represents the $\log _{10} \mathrm{IC}_{50}$ values for neutralisation curves. J. Broad-neutralisation of SARS-CoV, WIV16, RaTG13, SARS-CoV-2, SARS-CoV-2 Beta, SARS-CoV-2 Gamma, and SARS-CoV-2 Delta by T2_17. Sera post 14 days after four immunisation (bleed 4) was used for comparison. NISBSC standard for SARS-CoV-2 and SARS-CoV antiserum are used as reference. Mann-Whitney U demonstrated statistical significance (p-value: $* \leq 0.05, * *<0.01$, $* * * \leq 0.001)$.

Emergence of two human epidemics caused by ACE-2 using sarbecoviruses in past two decades highlights the urgent need for vaccines that can provide broad protection from SARS-

CoV-2 VOCs as well as to all the ACE-2 receptor using sarbecoviruses that have the potential

to spill-over from zoonotic animal reservoirs. To achieve pan-Sarbeco/pan-Beta coronavirus protection, various vaccine strategies have been employed such as mRNA expressing chimeric version of spike proteins from different coronaviruses ${ }^{26}$, as well as mosaic and cocktail nanoparticles expressing RBDs of different coronaviruses ${ }^{27}$. Though these strategies have been reported to be effective in generating pan-Sarbeco/pan-Beta coronaviruses immune responses, 
263 describe a new structural informatics platform pipeline for generating a single novel antigen

264 for sarbecoviruses, which generated broader responses to SARS-CoV, SARS-CoV-2 and

265 related bat sarbecoviruses. The informatics platform pipeline generates novel antigens as

266 synthetic genes allowing seamless down-stream immune screening of libraries of broadly

267 reactive vaccine antigens that can be compared and selected for optimal in vivo immune

268 responses by nucleic acid delivery. As proof of concept, lead pan-sarbecovirus vaccine antigen

269 candidates were immune selected for the broadest neutralisation profile, as confirmed in 3

270 species against SARS-CoV, WIV16, RaTG13, SARS-CoV-2 and it's VOCs. Further

271 broadening of vaccine protection across the betacoronavirus genus is being expanded using

272 combinations of multiple, digitally immune optimised antigens selected to immunologically

273 recruit additional $\mathrm{T}$ and $\mathrm{B}$ effector responses for maximising breadth and depth of immunity to

274 pre-emergent Coronaviruses. This approach is applicable for other complex and variable virus

275 families such as the Influenza viruses which represent current and future pandemic threats.

\section{Acknowledgements}

277 DBPR

\section{$278 \quad$ References}

279 1. Liu, K. et al. Cross-species recognition of SARS-CoV-2 to bat ACE2. Proc. Natl. Acad.

280 Sci. U. S. A. 118, (2021).

2. Olival, K. J. et al. Possibility for reverse zoonotic transmission of SARS-CoV-2 to freeranging wildlife: A case study of bats. PLOS Pathog. 16, e1008758 (2020).

3. Hu, B. et al. Discovery of a rich gene pool of bat SARS-related coronaviruses provides new insights into the origin of SARS coronavirus. PLOS Pathog. 13, e1006698 (2017).

4. Menachery, V. D. et al. A SARS-like cluster of circulating bat coronaviruses shows potential for human emergence. Nat. Med. 21, 1508-1513 (2015). 
5. Vilar, S. \& Isom, D. G. One Year of SARS-CoV-2: How Much Has the Virus Changed? Biology 10, (2021).

6. Horspool, A. M. et al. SARS-CoV-2 B.1.1.7 and B.1.351 variants of concern induce lethal disease in K18-hACE2 transgenic mice despite convalescent plasma therapy. BioRxiv Prepr. Serv. Biol. (2021) doi:10.1101/2021.05.05.442784.

7. Planas, D. et al. Sensitivity of infectious SARS-CoV-2 B.1.1.7 and B.1.351 variants to neutralizing antibodies. Nat. Med. 27, 917-924 (2021).

8. Wang, P. et al. Increased resistance of SARS-CoV-2 variant P.1 to antibody neutralization. Cell Host Microbe 29, 747-751.e4 (2021).

9. Leung, K., Shum, M. H., Leung, G. M., Lam, T. T. \& Wu, J. T. Early transmissibility assessment of the N501Y mutant strains of SARS-CoV-2 in the United Kingdom, October to November 2020. Euro Surveill. Bull. Eur. Sur Mal. Transm. Eur. Commun. Dis. Bull. 26, (2021).

10. Tian, F. et al. Mutation N501Y in RBD of Spike Protein Strengthens the Interaction 301 between COVID-19 and its Receptor ACE2. bioRxiv 2021.02.14.431117 (2021) doi:10.1101/2021.02.14.431117.

11. Campbell, F. et al. Increased transmissibility and global spread of SARS-CoV-2 variants of concern as at June 2021. Eurosurveillance 26, 2100509 (2021).

12. Wu, Y. et al. A noncompeting pair of human neutralizing antibodies block COVID-19 virus binding to its receptor ACE2. Science 368, 1274-1278 (2020).

307 13. Hwang, W. C. et al. Structural Basis of Neutralization by a Human Anti-severe Acute 308 Respiratory Syndrome Spike Protein Antibody, 80R. J. Biol. Chem. 281, 34610-34616 309 (2006).

310 14. Pinto, D. et al. Cross-neutralization of SARS-CoV-2 by a human monoclonal SARS311 CoV antibody. Nature 583, 290-295 (2020). 
312 15. Yuan, M. et al. A highly conserved cryptic epitope in the receptor binding domains of 313 SARS-CoV-2 and SARS-CoV. Science 368, 630-633 (2020).

314 16. Hatcher, E. L. et al. Virus Variation Resource - improved response to emergent viral $315 \quad$ outbreaks. Nucleic Acids Res. 45, D482-D490 (2017).

316 17. Li, W. et al. Animal Origins of the Severe Acute Respiratory Syndrome Coronavirus:

317 Insight from ACE2-S-Protein Interactions. J. Virol. 80, 4211-4219 (2006).

318 18. Lavie, M., Hanoulle, X. \& Dubuisson, J. Glycan Shielding and Modulation of 319 Hepatitis C Virus Neutralizing Antibodies. Front. Immunol. 9, 910 (2018).

320 19. Watanabe, Y. et al. Structure of the Lassa virus glycan shield provides a model for 321 immunological resistance. Proc. Natl. Acad. Sci. U. S. A. 115, 7320-7325 (2018).

322 20. Eggink, D., Goff, P. H. \& Palese, P. Guiding the immune response against influenza 323 virus hemagglutinin toward the conserved stalk domain by hyperglycosylation of the 324 globular head domain. J. Virol. 88, 699-704 (2014).

325 21. Schymkowitz, J. et al. The FoldX web server: an online force field. Nucleic Acids 326 Res. 33, W382-W388 (2005).

327 22. Nguyen, L.-T., Schmidt, H. A., von Haeseler, A. \& Minh, B. Q. IQ-TREE: A Fast and 328 Effective Stochastic Algorithm for Estimating Maximum-Likelihood Phylogenies. Mol. 329 Biol. Evol. 32, 268-274 (2015).

330 23. Ashkenazy, H. et al. ConSurf 2016: an improved methodology to estimate and 331 visualize evolutionary conservation in macromolecules. Nucleic Acids Res. 44, W344332 W350 (2016).

333 24. Schrödinger, L. \& DeLano, W. PyMOL. (2020).

334 25. Berman, H. M. et al. The Protein Data Bank. Nucleic Acids Res. 28, 235-242 (2000). 
26. Martinez, D. R. et al. Chimeric spike mRNA vaccines protect against Sarbecovirus

336 challenge in mice. BioRxiv Prepr. Serv. Biol. 2021.03.11.434872 (2021)

337 doi:10.1101/2021.03.11.434872.

338 27. Walls, A. C. et al. Elicitation of broadly protective sarbecovirus immunity by

339 receptor-binding domain nanoparticle vaccines. Cell (2021)

340 doi:10.1016/j.cell.2021.09.015.

341

342 Methods

\section{Phylogenetic analysis}

344 Protein sequences of spike proteins were downloaded from the NCBI virus database for all the

345 known sarbecoviruses. A multiple sequence alignment (MSA) was generated usingMUSCLE ${ }^{1}$.

346 The resulting MSA was pruned to the RBD region and used as input for phylogenetic tree

347 reconstruction. The phylogenetic tree was generated using IQTREE ${ }^{2}$ using the protein model

348 with the best BIC score. The resultant tree was used for generation of phylogenetically 349 optimised design using HyPhy ${ }^{3}$.

350

\section{$351 \quad$ Epitope identification}

352 Available structural data for spike protein-antibody complexes for SARS-CoV and SARS-

353 CoV-2 were downloaded from the Protein Databank (PDB) ${ }^{4}$. Structural data were then pruned

354 for antigen-antibody complexes where the epitopes were on the RBD. Amino acid residues of 355 antigen that have at least one atom within $5 \AA$ radii of at least one atom of amino acid of 356 antibody were defined as epitope residues, with epitope regions defined as contiguous stretches 357 of at least 5 amino acids. 
360 Structural models were generated for T2_13 using MODELLER ${ }^{5,6}$ using both SARS-CoV and

361 SARS-CoV-2 structures as templates. The structural model with the highest DOPE score ${ }^{7}$ was

362 chosen as the working model for further molecular modelling. The side chains for the model

363 were further optimised using $\mathrm{SCWRL}^{8}$ and energy minimised using GROMACS ${ }^{9}$. For T2-14

364 to T2-18, mutations were introduced using T2-13 as the reference structure using BUILD module of FOLDX algorithm ${ }^{10}$ and checked for structural stability using FOLDX forcefield ${ }^{10}$.

\section{Production and transformation of plasmids.}

368 Sequences of vaccine designs were gene-optimized and adapted to human codon use via the

369 GeneOptimizer algorithm ${ }^{11}$. These genes were cloned into pEVAC (GeneArt, Germany) via restriction digestion. Plasmids were transformed via heat-shock in chemically induced competent E. coli DH5 $\alpha$ cells (Invitrogen 18265-017). Plasmid DNA was extracted from transformed bacterial cultures via the Plasmid Mini Kit (Qiagen 12125). All plasmids were subsequently quantified using UV spectrophotometry (NanoDrop ${ }^{\mathrm{TM}}$-Thermo Scientific).

\section{Vaccination Experiments in Mice.}

Eleven groups of six female 8-10-week-old BALB/c mice were purchased from Charles River Laboratories (Kent, United Kingdom). Mice were immunised a total of four times with 30 days intervals. A total volume of $50 \mu 1$ of PBS containing $50 \mu \mathrm{g}$ of plasmid DNA was administered via subcutaneous route in the rear flank. Blood was sampled from the saphenous vein at 15 days intervals, and animals were terminally bled by cardiac puncture under non-recovery anaesthesia at day 150 . 
HEK293T cells were transfected with an expression plasmid expressing wild-type spike glycoprotein of each of the four ACE-2 binding sarbecoviruses including SARS-CoV, SARS-CoV-2, RaTG13, and WIV16. 48 hours after transfection, cells were transferred into V-bottom 96-well plates (50,000 cells/well). Cells were incubated with sera (diluted at 1:50 in PBS) or anti-mouse IgG Isotype negative control (Invitrogen $10400 \mathrm{C}$, diluted at $20 \mu \mathrm{g} / \mathrm{mL}$ in PBS) for 30 min, washed with FACS buffer (PBS, 1\% FBS, 0.02\% Tween 20) and stained with Goat anti-mouse IgG (H+L) Alexa Fluor 647 Secondary Antibody (Invitrogen A32728, diluted at $20 \mu \mathrm{g} / \mathrm{mL}$ in FACS buffer), for 30 min in the dark. Cells were washed with FACS buffer and samples were run on a Attune NxT Flow Cytometer (Invitrogen) with a high-throughput auto sampler. Dead cells were excluded from the analysis by staining cells with 7Aminoactinomycin D (7-AAD) and gating 7-AAD negative live cells.

\section{Enzyme-linked immunosorbent assay (ELISA)}

397 The assays were adapted from those originally described by Amanat and co-workers ${ }^{12}$. Briefly, Nunc MaxiSorp ${ }^{\mathrm{TM}}$ flat-bottom plates were coated with $50 \mu \mathrm{l}$ per well of $1 \mu \mathrm{g} / \mathrm{ml}$ of RBD from SARS-1 or SARS-2 DPBS $\left(-\mathrm{Ca}^{2+} /-\mathrm{Mg}^{2+}\right)$ and incubated overnight at $4{ }^{\circ} \mathrm{C}$. The next day, the 400 plates were blocked with 3\% milk in PBST $(0.1 \% \mathrm{w} / \mathrm{v}$ Tween20 in PBS) for 1 hour. After 401 removing the blocking buffer, $50 \mu \mathrm{l} /$ well of serum samples diluted in PBST-NFM $(1 \% \mathrm{w} / \mathrm{w}$ non-fat milk in PBST) were added to the plates and incubated on a plate shaker for two hours at $20^{\circ} \mathrm{C}$. The plates were washed three times with $200 \mu \mathrm{l}$ of PBST, and then $50 \mu \mathrm{l}$ of HRPconjugated goat anti Ig (H and L chains) (Jackson ImmunoResearch) was added to each well and left to incubate for one hour on a plate shaker for 1 hour. Plates were washed three times with $200 \mu 1$ of PBST, $50 \mu 1$ /well of 1-Step Ultra TMB chromogenic substrate (Sigma) was added to the plates and the chemical reaction was stopped three minutes later with $50 \mu 12 \mathrm{~N} \mathrm{H}_{2} \mathrm{SO}_{4}$. 
408 The optical density at a wavelength of 450nm (OD450) was measured using a BioRad 409 microplate reader. Values from the dilution curve were used to determine the area under the 410 curve.

412 Pseudotype-based micro-neutralisation assay

413 Pseudotype-based micro-neutralisation assay was performed as described previously ${ }^{13}$. 414 Briefly, serial dilutions of serum were incubated with SARS-CoV-2/RaTG13/SARS$415 \mathrm{CoV} / \mathrm{WIV} 16$ spike bearing lentiviral pseudotypes for $1 \mathrm{~h}$ at $37^{\circ} \mathrm{C}, 5 \% \mathrm{CO}_{2}$ in 96 -well white 416 cell culture plates. $1.5 \times 10^{4}$ HEK293T/17 transiently expressing human ACE-2 and TMPRSS2 417 were then added per well and plates incubated for $48 \mathrm{hrs}$ at $37^{\circ} \mathrm{C}, 5 \% \mathrm{CO}_{2}$ in a humidified 418 incubator. Bright-Glo (Promega) was then added to each well and luminescence read after a 419 five-minute incubation period. Experimental data points were normalised to $100 \%$ and $0 \%$ 420 neutralisation controls and non-linear regression analysis performed in GraphPad Prism 9 to 421 produce neutralisation curves and $\mathrm{IC}_{50}$ values.

\section{Vaccine boost efficacy studies in K18-hACE2 mice.}

424 Ten groups of six female 8-15-week-old homozygous K18-hACE2 mice (Jax) were primed 425 with $1.4 \times 10^{9}$ viral particles of AZD1222 or PBS by intramuscular route, in a total volume of 426 $100 \mu 1$ split over the two rear legs. After 28 days, two groups of six mice were boosted with PBS, AZD1222, or T2_17 DNA. Mice were bled at two-week intervals and challenged at day 84 with either Victoria/1/2020 (B-type) or Delta SARS-CoV-2 by intranasal route, in a total volume of $40 \mu 1$ over both nares. Mice were weighed daily and monitored for clinical signs for a period of 10 days before being culled by terminal bleed. 
Intradermal nucleic acid immunisation with Tropis PharmaJet delivery in Guinea pigs.

433 Two groups of eight female 7-week-old Dunkin Hartley Guinea pigs (Envigo RMS, 434 Blackthorn, United Kingdom) were immunised a total of three times with 28 days intervals. A 435 total volume of $200 \mu 1$ of PBS containing $400 \mu \mathrm{g}$ of plasmid DNA was administered by 436 PharmaJet Tropis intradermal device, split over each hind leg. Blood was sampled from the saphenous vein at 14 days intervals.

Intradermal nucleic acid immunisation with Tropis PharmaJet delivery in in Rabbits.

440 Ten mature (five male, five female) rabbits were immunised with a GMP lot pEVAC_T2_17

441 (clinical pEVAC_PS) intradermally by PharmaJet Tropis needleless delivery to the upper left 442 and right hind limbs $(300 \mu \mathrm{l}$ at $2 \mathrm{mg} / \mathrm{mL})$. For control group, ten mature (five male, five female) rabbits were injected with PBS. Arterial blood was sampled at 14 days intervals

\section{ACE-2 competition assay}

The SARS-CoV-2 surrogate virus neutralisation test (SVNT, Genscript, Piscataway, New Jersey, United States) was carried out as per manufacturer's instructions. Briefly, serum from bleed 6 guinea pigs were diluted in PBS across an 8 point 1:2 dilution series from a starting concentration of 1:50. Samples were further diluted in the provided sample buffer at a 1:9 ratio, and then mixed with HRP conjugated to SARS-CoV-2 RBD protein, incubated at $37^{\circ} \mathrm{C}$ for 30 min and added to human ACE-2 protein coated wells in 96-well plate format. The reaction was incubated at $37^{\circ} \mathrm{C}$ for $15 \mathrm{~min}$ and then washed four times with provided wash buffer. TMB solution was then added, incubated for 15 minutes in the dark at R.T to allow the reaction to develop. The reaction was then quenched using the provided stop solution, and then absorbance read at $450 \mathrm{~nm}$. 
458 Mann-Whitney U tests were performed for all the comparisons using the Python sklearn package $^{14}$. All the plots were generated using the Python Matplotlib package ${ }^{15}$.

460

\section{References}

462

1. Edgar, R. C. MUSCLE: a multiple sequence alignment method with reduced time and space complexity. BMC Bioinformatics 5, 113 (2004).

465

2. Nguyen, L.-T., Schmidt, H. A., von Haeseler, A. \& Minh, B. Q. IQ-TREE: A Fast and 466 Effective Stochastic Algorithm for Estimating Maximum-Likelihood Phylogenies. Mol. 467 Biol. Evol. 32, 268-274 (2015).

3. Pond, S. L. K., Frost, S. D. W. \& Muse, S. V. HyPhy: hypothesis testing using phylogenies. Bioinformatics 21, 676-679 (2005).

4. Berman, H. M. et al. The Protein Data Bank. Nucleic Acids Res. 28, 235-242 (2000).

5. Eswar, N. et al. Comparative protein structure modeling using MODELLER. Curr. Protoc. Protein Sci. Chapter 2, Unit 2.9 (2007).

6. Sali, A. \& Blundell, T. L. Comparative protein modelling by satisfaction of spatial restraints. J. Mol. Biol. 234, 779-815 (1993).

7. Shen, M. \& Sali, A. Statistical potential for assessment and prediction of protein structures. Protein Sci. Publ. Protein Soc. 15, 2507-2524 (2006).

8. Krivov, G. G., Shapovalov, M. V. \& Dunbrack, R. L. Improved prediction of protein sidechain conformations with SCWRL4: Side-Chain Prediction with SCWRL4. Proteins Struct. Funct. Bioinforma. 77, 778-795 (2009).

9. Van Der Spoel, D. et al. GROMACS: fast, flexible, and free. J. Comput. Chem. 26, 1701481 1718 (2005). 
482 10. Schymkowitz, J. et al. The FoldX web server: an online force field. Nucleic Acids 483 Res. 33, W382-W388 (2005).

484 11. Raab, D., Graf, M., Notka, F., Schödl, T. \& Wagner, R. The GeneOptimizer 485 Algorithm: using a sliding window approach to cope with the vast sequence space in 486 multiparameter DNA sequence optimization. Syst. Synth. Biol. 4, 215-225 (2010).

487 12. Amanat, F. et al. A serological assay to detect SARS-CoV-2 seroconversion in 488 humans. Nat. Med. 26, 1033-1036 (2020).

489 13. Carnell, G., Grehan, K., Ferrara, F., Molesti, E. \& Temperton, N. J. An Optimised 490 Method for the Production using PEI, Titration and Neutralization of SARS-CoV Spike 491 Luciferase Pseudotypes. Bio-Protoc. 7, (2017).

492 14. Pedregosa, F. et al. Scikit-learn: Machine Learning in Python. J. Mach. Learn. Res. $493 \quad \mathbf{1 2}, 2825-2830(2011)$.

494 15. Hunter, J. D. Matplotlib: A 2D Graphics Environment. Comput. Sci. Eng. 9, 90-95 495 (2007).

496 


\section{Supplementary Files}

This is a list of supplementary files associated with this preprint. Click to download.

- 20210914222T217supplfinal260921.pdf

- rs.pdf 